\title{
ANÁLISIS DEL CONCEPTO DE SOCIABILIDAD EN LAS CIENCIAS SOCIALES
}

\author{
Analysis of the concept of sociability in social sciences
}

\author{
Daniel Roberto Vega Torres \\ Fundación Universitaria Juan de Castellanos, Colombia, daniel.vega@uptc.edu.co
}

Recibido: 5 de febrero, 2015 Aceptado: 24 de noviembre, 2015

\begin{abstract}
Resumen: El objetivo del artículo es analizar el concepto de sociabilidad como una propuesta teórica social que permite comprender las formas de asociación, al tener en cuenta sus aportes y alcances para las ciencias sociales. El análisis está dividido en dos temáticas: la primera sociológica, donde se analiza el concepto como una forma específica de socialización en la teoría de Georg Simmel y la propuesta funcionalista de Georges Gurvitch como parte de la microsociología; la segunda desde la historia de las asociaciones en la teoría de Maurice Agulhon, como base del conocimiento de lo cotidiano. Se reconoce una discontinuidad en las propuestas y en los significados del concepto durante el siglo XX. Se considera como aporte al ser una forma más abierta de comprender las asociaciones, pues no presupone un estado homogéneo de clasificación social, sino que permite obtener representaciones diversas. Se propone que sus límites se encuentran tanto en el análisis de la forma estructural como en la intencionalidad de los sujetos.
\end{abstract}

Palabras clave: Sociabilidad, Sociología, Historia.

\begin{abstract}
The aim of this paper is to analyze the concept of sociability as a theoretical social proposal that allows for the understanding of the different types of associations by taking into consideration its contributions and scopes in the social sciences. The analysis is divided into two approaches: the first one is a sociological approach, in which sociability is analyzed as a specific form of socialization in Georg Simmel's theory and Georges Gurvitch's functionalist proposal as part of microsociology; the second approach analyzes the concept from the history of associations in Maurice Agulhon's theory as the basis for everyday knowledge. Discontinuity is found in the proposals and in the meanings of the concept during the twentieth century. This paper is a contribution to sociability because it is a more open way to understand associations, since it does not assume a homogeneous state of social classification, but rather allows for different representations. It is proposed that its boundaries are the analysis of structural form and the subjects' intent.
\end{abstract}

Keywords: Sociability, Sociology, History.

\section{Introducción}

Actualmente, los trabajos sobre sociabilidad han venido en aumento en Latinoamérica, en donde se tienen varios intereses de análisis como la transformación laboral y espacial (Marques, 2011; 
Bayón, Saraví \& Ortega, 2013; White, 2013) y la historia de las asociaciones de los siglos XVIII y XIX (Goicovic, 2005; Chapman, 2008; Guarín, 2010; Loaiza, 2011). Este recurso conceptual adquiere relevancia en formas de organización social actual, en especial desde la ampliación tecnológica de mecanismos y medios de relación (Lash, 2003). El objetivo de este texto es analizar el concepto de sociabilidad como una propuesta teórica social que permite comprender las formas de organización de la vida en sociedad, y tener en cuenta sus aportes y alcances para las ciencias sociales.

González (2008) mencionó dos formas para el análisis de este concepto: la sociabilidad como categoría histórica y como categoría analítica. La primera en referencia a las formas de interpretar la sociabilidad como un contenido histórico desde los significados en cada época o lugar, mientras que la categoría analítica se consideraba como objeto epistemológico de la historia. Así, desde esta clasificación, se presenta un desarrollo de la sociabilidad como un espacio de debate sobre la construcción del pensar socio-histórico. El análisis está dividido en dos temáticas, la primera sociológica y la segunda histórica.

Como un primer acercamiento desde lo sociológico, se expone de manera general la forma en que Georg Simmel (1858-1918) presentó este concepto para entender una forma de socialización y desde allí explicar un ejemplo de la sociología formal. El segundo acercamiento considera la obra de Georges Gurvitch (1894-1965) respecto a las formas de sociabilidad, es decir, un cambio de paradigma de lo que es la "estructura social", entendida como la manera en y por lo que se relacionan los individuos. Luego se realiza un énfasis histórico, que parte de la apropiación del concepto en los trabajos de Maurice Agulhon (1926-2014) como una categoría histórica en crítica al concepto de "mentalidades". Por último se hace una reflexión del concepto que reconoce algunas formas metodológicas de aplicación y sus límites en los estudios sociales.

\section{Sociabilidad como forma de socialización}

La sociabilidad como categoría relacional se manifiesta en el debate y surgimiento de lo sociológico durante el siglo XIX y comienzos del XX, en la obra del sociólogo alemán Simmel (1917), quien se distancia de los planteamientos de los pensadores como Marx y Durkheim y produce un aporte autónomo y original sobre la cuestión. Una de las preguntas que gobierna el trabajo teórico de la sociología en Georg Simmel es ¿cómo es posible la sociedad? Parte de la realidad social como algo distinto a una realidad natural. Pues, según Kant, mientras la "naturaleza" se objetiva en el sujeto cognoscente, haciéndose"natural" por el hecho mismo de darle orden un sujeto"contemplador"; lo social implica -para Simmel- una unidad a priori que no requiere de contemplador, que se encuentra fuera del límite del yo cognoscente y trasciende al ámbito de la existencia misma del ser social o ser-en-relación; lo cual responde, de esta manera, a las"condiciones, puestas a priori en los elementos mismos, gracias a las cuales se unen estos realmente para formar la 'sociedad" (Simmel, 2002b, p. 77). Así, la sociología comprende los procesos que condicionan el 
devenir de lo social o "socialidad" (Gesellschafts-Sein), ${ }^{1}$ entendido este como un proceso que lleva a la síntesis denominada "sociedad". De allí que ésta no sea algo externo y abstracto, sino que se comprenda como algo que los individuos hacen y sufren, por ello para Simmel sería mejor hablar de socialización (Vergesellschaftung), que de sociedad (Gesellschaft) (Simmel, 2002a, p. 33).

Para Simmel la sociología es el estudio de las condiciones o formas del proceso de socialización, que se sustentan en la interacción o acción recíproca. La idea de sociedad como una suma de elementos se deja a un lado, para comprender que los demás no son "individuos", pues en la acción recíproca no vemos individualidades, sino generalidades del otro respecto a su profesión, actividad o círculo de pertenencia (militar, religioso, profesor, etc.). No vemos a los demás puramente como individuos, sino como colegas, compañeros, o correligionarios; en una palabra, como habitantes del mismo mundo en particular. Y este supuesto inevitable, que actúa de un modo automático, es uno de los medios que tiene el hombre para dar a su personalidad y realidad, en la representación del otro, la cualidad y formas requeridas por su sociabilidad (Simmel, 2002b, p. 83).

Las formas de socialización, como formas de pertenencia a grupos que permiten la socialidad, también generan un grado de "insocialidad". Pues el sujeto no se limita a su definición dentro de una sociedad, organización o profesión, depende de ámbitos sociales y "no-sociales". "El elemento 'no-social' recoge por completo la personalidad, con su color especial, con su irracionalidad y su vida interior" (Simmel, 2002b, p. 85). Esta tensión es la que desarrolla el proceso de socialización, pues el individuo no solo se encuentra dentro de la sociedad, sino al mismo tiempo fuera de ella. Estos no son "momentos", sino características del ser social, entendido este como categoría sintética, producto y miembro a la vez, de la vida social. Al hablar de sociedad no se concibe la homogeneidad, sino lo heterogéneo, una síntesis de desigualdades.

Pero, entonces, ¿cuáles serían las formas de comprensión de la vida social desde un estudio sociológico? Simmel presenta tres problemas principales en la Sociología: El primero es el estudio sociológico de la vida histórica, es decir, la manera como las sociedades configuradas históricamente pueden estudiarse mediante leyes o generalidades. El segundo problema es el estudio propiamente de las formas de socialización, es el devenir mismo de las relaciones recíprocas que tienden a la configuración de la vida social. Por último, una sociología filosófica que pretende abarcar el ámbito gnoseológico y metafísico, destinado a las preguntas trascendentes de lo social, como su finalidad y sentido (Simmel, 2002b, pp. 42-55). Este estudio se orienta en el segundo problema, sobre la forma de socialización.

La socialización es la forma en que se produce la práctica recíproca o interacción entre los individuos, es decir la manera como se influencian unos con otros para constituir unidades de diverso grado (familias, Estado, partido, etc.), esta surge por determinados fines o motivaciones

$\overline{1}$ Se incluyen los conceptos en alemán y francés para indicar su diferencia en cada contexto. 
(políticos, económicos, religiosos, afectivos, etc.) que se crean en la materia o contenido de la socialización. Estas motivaciones, fines o intereses no constituyen la socialización en sí mismas, sino solo cuando accionan recíprocamente o intentan influir en el otro. Para Simmel existen diversas formas de socialización como la lucha, el intercambio, la dominación y hasta la prostitución. No obstante, hay una forma de socialización particular que lleva in nuce la crítica a los planteamientos epistemológicos de la sociedad, la sociabilidad.

Las formas en que se desarrolla la vida social pueden llegar a ser autónomas, liberadas de su carácter teleológico o útil. En ese sentido, la sociabilidad (Geselligkeit) como forma de socialización (Vergesellschaftungform) es su manifestación más abstracta o lúdica; es el fenómeno por el cual se despoja de todo aspecto material la vida social, el interés por las personalidades queda relegado al estar juntos. Es el mundo artificial (künstliche Welt) por el cual se pretende la igualdad entre los miembros, en donde se depende del ánimo del individuo y el de los otros. Según Simmel, la sociabilidad puede pensarse como la abstracción de la socialización, que tiene un carácter de juego y puede presentarse como la más transparente de las relaciones sociales, pues se realizaría entre personas iguales².

Este tipo de abstracción de la socialización propende por una separación de lo "serio" y del "juego", una realidad "real" (ética) y una realidad "artificial" (estética). La primera convendría más al trabajo, a las formas de competencia e intercambio económico; la segunda, a la estilización de la vida, al puro acontecer de la relación en un ámbito de diversión (lúdico). No obstante, la separación entre los dos tipos de realidades (esencial/aparente) mantendrá una división epistémica que limita el argumento de la sociabilidad a formas "superficiales" de lo real. Pero, si se interpreta esta "superficialidad" (Oberflächlichkeit), no como un detrimento de "lo real" en la vida social, sino como apariencia (Sheine), es decir, se comprende que la vida en sociedad es un juego simbólico (Symbolisches Spiel) que sabemos que estamos jugando ${ }^{3}$, el resultado es distinto, pues nos propone de manera general repensar el planteamiento de la sociabilidad como una representación estética de las formas de vida.

\section{Sociabilidad como aspecto microsociológico}

Luego de la comprensión de la sociabilidad (Geselligkeit) como el aspecto abstracto de la socialización en Simmel, pasamos a una forma de comprensión de lo social que desarrolla en la

2 En el texto original se encuentra la siguiente oración: "Sie ist das Spiel, in dem man »so tut", als ob alle gleich wären, und zugleich, als ob man jeden besonders ehrte" (Simmel, 1917, p. 61)

3 Recordemos que uno de los aportes teóricos a la teoría de Simmel es la obra de F. Nietzsche, pues para el primero, el "misterio de la vida" o la liberación de la vida ocurre en la configuración y experimentación "en el juego autónomo de sus formas el sentido y las fuerzas de su realidad más profunda, pero sin esta realidad misma", lo que mutatis mutandis se entendería a la vida como la apariencia, como el juego o el sueño, es para Nietzsche la "conciencia de la apariencia" (Das Bewusstsein vom Scheine), ese soñar sabiendo que se sueña con otros (Träumenden unter einander), describiendo dicho filósofo en su Aforismo 54 de la Gaya Ciencia, "Para mí, la apariencia es la viva realidad misma actuando, que, irónica consigo misma, había llegado a hacerme creer que aquí no hay más que apariencia, fuegos fatuos, danzas de duendes y nada más, así como que entre todos esos soñadores también yo, atravesando un 'trance de conocer', bailo mi propia danza". 
sociabilidad (Sociabilité) sus formas o tipos. Georges Gurvitch presenta una división del estudio de la "Sociología general" en macrosociológica y microsociológica, se basa en la comprensión de la realidad desde los componentes más simples a los más complejos. Frente a la sociología moralista y teleológica que se encontraba a finales del siglo XIX, (Gurvitch, 1938/1941) propone el "empirismo sociológico radical", que se fundamenta en el pluralismo, en la transposición de diversas formas de sociabilidad o componentes de asociación en una misma realidad y tiempo, y que, consecuente a ello, no jerarquiza ninguna de esas relaciones, sino que las propone como diferenciación de los enfoques conceptuales simple y complejo. Esta diferenciación se basa en el significado de "estructura social" que puede entenderse según el autor en tres formas:

1. Sea los tipos de unidades colectivas particulares: agrupaciones de actividad, de localidad, de parentesco, de amistad, de adoración, etc., en la variedad casi infinita de sus subtipos, de sus entrecruzamientos y de sus conflictos complejos (estructura de las agrupaciones); 2 . Sea los tipos históricos de su combinación, de su integración y su desintegración en la sociedad global (estructura de la sociedad global en tal o cual época), 3. sea, en fin, los tipos de la sociabilidad misma, es decir, las diferentes maneras de estar ligados en un todo y por un todo social (Gurvitch, 1938/1941, p. 20).

Las primeras dos corresponden a lo que sería el ámbito macrosociológico, es decir la unidad colectiva o agrupación y la sociedad global. El tercer ámbito, las formas de sociabilidad, se presentan como elementos constitutivos de la vida social, aquellos que se considerarían parte indivisible de la realidad social. Desde la comprensión de la sociabilidad se encuentra, no ya la manera como se focaliza un hecho, individuo o actividad, sino que se reflexiona la forma en que se afectan mutuamente los elementos de la realidad social. Estos elementos constitutivos por una "conciencia colectiva"4 permiten diferenciar dos formas de manifestación de la sociabilidad: una directa (o espontánea) y otra reflexiva (u organizada).

La sociabilidad espontánea puede darse por simple convergencia o de manera "interpenetrada" (interpénétrée) o de afectación mutua, es decir, por el estado de conciencia colectiva que puede ir de un grado máximo de afectación mutua del "Nosotros" a la interdependencia por simple convergencia de un "Yo" con los "Otros". Ese "Nosotros", que implica una unidad, se divide según la intensidad de la misma de mayor a menor en comunión, comunidad y masa. El criterio que se utiliza para medir el grado de relación de la conciencia colectiva es la intensidad (intensité), así: "las relaciones de acercamiento, en que la intensidad de la convergencia es más fuerte, las relaciones de alejamiento (luchas, conflictos) en que es la menor, y las relaciones mixtas (acercamiento que provoca alejamiento) en que es media" (Gurvitch, 1938/1941, p. 32).

La sociabilidad organizada no presenta una presión, sino una coacción entre las superestructuras organizadas y las infraestructuras espontáneas. Al converger con las formas es-

4 Retomando el planteamiento de Durkheim, Gurvitch define que "la conciencia colectiva está en cada uno de nosotros y cada uno de nosotros está en la conciencia colectiva". (Gurvitch, 1938/1941, p. 29). 
pontáneas en las unidades colectivas, presentan la misma diferenciación de un "Nosotros" (masa, comunidad y comunión); puede decirse que es, en cierta medida, más trascendente o de orden institucional. En esta medida, las formas básicas de toda institución, o mejor, de ese "Nosotros institucionalizado" se presentan en la colaboración y en la dominación.

La sociabilidad espontánea estaría ligada a procesos más dinámicos por presión, mientras que la organizada tiende más a la reproducción e institucionalización por coacción. Las dos formas o criterios tienen en común el hecho de distinguirse internamente entre una sociabilidad activa y una pasiva. La primera persigue una obra común que permite la construcción de superestructuras organizadas, mientras que la otra no. Así, el cruce de estos tipos de categorización conformará un panorama de las posibilidades de vivir en sociedad, las formas de sociabilidad. La presentación de las formas de relación de estos elementos puede ser, para Gurvitch, infinita.

La diferenciación de las formas de sociabilidad se medirá en grados de intensidad o afectación mutua que posea una unidad o conciencia colectiva, valor que es inverso al grado de presión. Por ejemplo, la masa se presenta como un "Nosotros" con un bajo grado de "interpenetración" y un mayor estado de presión. De forma contraria la comunión presenta una alta intensidad y una baja presión. De manera similar a Simmel, se le da a ese "Nosotros" una forma preexistente, como el idioma. Lo significativo de Gurvitch es que no toma los planteamientos de Simmel para configurar las formas de sociabilidad, sino que se basa en los trabajos de Emilie Durkheim, Max Scheler, Ferdinand Tönnies y Leopold Von Wiese. Así, este autor franco-ruso replantea la sociología desde la perspectiva funcionalista, pasando de una física social evolucionista a una física social pluralista de la realidad.

El aporte de este autor fue el de consolidar la sociabilidad como un aspecto constitutivo de la sociedad, que merece un estudio particular, pues a diferencia del planteamiento de Simmel que lo considera como un caso extremo de la abstracción de la socialización, este autor lo pone como base de la concepción ontológica de lo social. No es ya una pregunta por una forma de comprender las relaciones sociales (epistemología), sino la manera como el ser social vive en sociedad, se relaciona y constituye formas de vida plurales, diversas y contradictorias en una misma realidad. No hay continuidad en el estudio, sino más bien un encuentro de dos autores, uno por la comprensión de la socialización y otro por la reinterpretación de la conciencia colectiva, es decir otra manera de comparar el vitalismo y el funcionalismo, respectivamente.

Aunque los aportes de Gurvitch se retomarán en la obra de historiadores franceses que consolidan el estudio en la segunda mitad del siglo XX, es importante resaltar que en relación con el estudio de la historia de los Annales, este autor tuvo una fuerte crítica frente a este tipo de "modelos" de explicación social, o al menos, de esta forma Braudel intenta denominar a este autor como alguien que adora destruir, ya que su planteamiento deja a un lado la historia y se enfoca en un tipo de estudio abstracto, su teoría es fragmentaria, discontinua (Braudel, 1953). Sin embargo, existirán otros historiadores franceses, como Maurice Agulhon, quienes retomarán sus ideas principales y las aplicarán como categoría histórica. 


\section{Sociabilidad e historia de las asociaciones}

La problemática por el ser social en Sociología termina por tomar un rumbo concreto en el análisis de la historia de la sociabilidad en la segunda mitad del siglo XX. Es el trabajo de Maurice Agulhon, en su estudio de las formas de las mentalidades colectivas (mentalités collectives) de la Francia de la primera mitad del siglo XIX, que logró consolidar con el libro de 1977, Le Cercle dans la France bourgeoise, 1810-1848. El aporte que este libro trae es la manera como la mentalidad colectiva, concepto histórico que deriva de la historia de las ideas, cambia hacia formas concretas de la vida social que recurren, no solo a las representaciones colectivas, sino que se nutre de la manera como se hace sociedad en las acciones cotidianas. La sociedad es una realidad histórica y su estudio comprende aspectos tanto de la vida privada y pública, como de sucesos, acciones y proyecciones de la vida social de grupos concretos, determinados y definidos.

El trabajo de Agulhon se ha considerado paradigmático en la formulación del estudio de la sociabilidad como categoría histórica debido a su aporte en la sistematización de un estudio social que escapaba de la dinámica tradicional de la historia de los Annales en su pretensión de totalidad, y se concentraba en aspectos de la vida de las asociaciones, sean estas burgueses, populares, laicas o religiosas 5 . Según Michel Vovelle es una nueva visión de la historia de las mentalidades, pues aunque todavía lo considera parte de la explicación estructural de las clases sociales, menciona que en su trabajo de 1966 revela aspectos de gran originalidad en el análisis histórico, como la vida de las asociaciones y la presencia de una vida municipal estructurada (Vovelle, 1967, p. 51). Ese estudio de la vida en sociedad es lo que diferencia a los trabajos de la búsqueda de lo imaginario y de la mentalidad.

La sociabilidad se diferencia de la historia de las mentalidades en retomar la importancia de la vida cotidiana como un aspecto conjunto de la realidad, es decir, se relaciona más con lo que él denominará como "historia de las asociaciones", en donde el papel del territorio tiene una gran relevancia en la forma de "densidad", que acompañaría el término de "intensidad" en un panorama espacial, es decir, masa y fuerza de la misma creada por la relación social o asociación. La falta de un desarrollo de este estudio se concentraba, según Agulhon, en la manera como se reproducía el marxismo y el funcionalismo en Francia durante esa época, pero al retomar los planteamientos de Max Weber, en donde se toma a su obra como una"sociología de las asociaciones", pero se tiene la precaución de no caer en anacronismos.

Para Agulhon, el estudio de las sociabilidades es "de algún modo, la historia conjunta de la vida cotidiana, íntimamente ligada con la psicología colectiva" (Agulhon, 1977/2009, p. 38). Es decir, identificar en las asociaciones, las actividades, lugares, normas, tiempos y recursos con los cuales se puede crear una práctica social continua. La idea de que la vitalidad de las asociaciones

5 Un ejemplo de ello es la obra Histoire vagabonde, en donde se presenta el estudio de asociaciones populares como la Chambrée, Cabarets, Goguettes, Societés, entre otros. (Agulhon, 1988/1994). 
es un buen indicador de la sociabilidad general de una colectividad humana no debería dar lugar a objeciones. Cuanto más numerosas y diversas son las relaciones interpersonales, más grupos se ponen en juego: la familia, la parroquia, el trabajo o el grupo de edad son una suerte de mínimo encuadre, al que vendrán a agregarse, o no, el partido político, el club deportivo, la sociedad de beneficencia, o lo que pueda imaginarse.

Por otro lado, cuantas más actividades tiene una asociación, más requiere fortalecer su organización interna (Agulhon, 1988/1994, p. 39), en otras palabras, desarrollar una serie de normas y consensos que sirvan para controlar y darle continuidad. Siguiendo a Agulhon, la manera como debe estudiarse una asociación no es mediante la fragmentación como lo propone la historiografía tradicional, en donde se analiza lo político, lo religioso, lo cultural y lo cotidiano separadamente, como si fueran categorías abstractas de la realidad social. Por lo tanto estudiar una asociación requiere entender que las relaciones sociales comprenden diversas series de categorías, que la complejidad de las situaciones asociativas implica valores, normas y escenarios contemplados según los intereses y actividades particulares de los individuos que conforman la asociación. La manera como devienen las asociaciones está dada en su aparición cada vez más numerosa y diversificada, y a la vez el grado de institucionalización o formalización que se consiga, en otras palabras el paso de un estadio informal a uno formal.

El trabajo sobre los círculos burgueses de Agulhon puede entenderse como un estudio sociológico e histórico (Pontón, 1980, pp. 12-77), que no se enfocaba en aspectos cristalizados, sino en las relaciones y mutaciones de las asociaciones según las proyecciones, intereses y recursos que disponían sus integrantes. Aunque el trabajo histórico no presenta una categorización clara de la sociabilidad política burguesa, si se puede encontrar un asidero en los trabajos de Agulhon para la interpretación de lo que es la sociabilidad en trabajos siguientes tanto en Europa (Boutier, 1994; Boutry, 1996) como en Latinoamérica (Zambrano, 1987; Guerra, 1992; González, 1999).

\section{Análisis de la sociabilidad}

Reflexionar la sociabilidad como categoría de las ciencias sociales y su uso analítico e histórico permite observar la manera como un conjunto de esfuerzos explicativos de las relaciones sociales contribuye a delimitar proyectos, formas y alcances de la investigación social. Así, desde las propuestas formales de Simmel, que son un tipo concreto de relación social, pasando por los aportes de Gurvitch, que son un análisis microfísico y funcional de la integración social, y con la propuesta histórica de Agulhon para entender relacionamientos sociales en espacios específicos de grupos política y económicamente diferenciados, se encuentra una discontinuidad en la aplicación y sentido del concepto, pero mantienen la idea principal de convertir a este en una herramienta de análisis de las formas de asociación como base de lo social.

En este sentido, mientras que las condiciones sociales permiten una interpretación y dinámicas cambiantes sobre la manera de su organización, la correspondencia en los estudios de la sociabi- 
lidad puede contribuir como un escenario de discusión de la manera como se explica la actividad social en cualquier escala. Además de ser una categoría que imprime el esfuerzo analítico en la interacción en un espacio, puede ser una herramienta elemental para el diagnóstico, como es el caso de su aplicación en el estudio analítico de las redes sociales (ARS) o Social Network Analysis.

El objetivo de la ARS es estudiar cómo se relacionan los individuos o agentes y qué estructura social se produce por dicha interacción para dar como resultado un tipo de normas. Los componentes principales son los siguientes: los agentes y sus acciones de manera interdependiente; actor, lazo relacional de agentes, díada, tríada, subgrupo, grupo, relación de grupos y red social (Wasserman \& Faust, 1994, pp. 17-20). Por un lado, se postula que todo actor está relacionado por lazos con otros agentes, lo que permite que según la cantidad (díada, tríada, subgrupo o grupo) puede conformar estructuras que igualmente se relacionan e interaccionan. Por otro lado, una red social consiste en el conjunto de agentes y relaciones entre ellos.

También, como aportes teóricos a las ciencias sociales, se obtiene una forma más abierta de comprender las asociaciones, pues no presupone un estado homogéneo de clasificación social, sino que permite obtener representaciones diversas (Forse, 1981; Bidart, 1988), con las siguientes variables:

1. La integración (intensidad), o el grado de coacción o fuerza que ejerce una asociación, esta se visualiza mediante el número de participantes, la continuidad de los mismos en la organización, la frecuencia de su trato, esto se complementa con la disposición de actores externos en relación con la colectividad o asociación.

2. La institucionalidad, el grado de formalidad u organización que permite visualizarse mediante las normas, códigos y actividades debidamente sistematizadas para el desarrollo de una práctica continúa. En este caso se considera que las formas de institucionalización dependen del grado de interiorización de dichas normas y códigos en los actores para el desarrollo de las actividades.

3. La duración o vitalidad de la asociación, pues permite establecer el tiempo que tuvo la asociación y su continuidad. En este caso, el tiempo debe entenderse, más que un segmento lineal, como una construcción social que tiende a imponer un orden secuencial.

4. El espacio social, que es aquel lugar o mecanismo que permite un desarrollo fluido de relaciones sociales, sustentadas principalmente por la rutina y la cotidianidad de las labores internas realizadas por un grupo o comunidad.

5. La comunicación o el grado de relación que se observa entre los miembros de la asociación y agentes externos a ella, que hacen uso de símbolos, códigos o narraciones, tanto en el papel de receptores, como de productores. 
6. No obstante, las limitantes del estudio de la sociabilidad son evidentes en especial por dos razones: primero, se parte de la idea de relación social como un fenómeno formal, donde permite que las condiciones culturales, económicas y ambientales y los conflictos que estas originan puedan presentarse ocultas y hasta invisibles debido al uso de datos relacionales sin atributos, lo que compromete negativamente al análisis de la realidad social. La sociabilidad se presenta, entonces, como un esfuerzo histórico, social y analítico de cómo deviene lo social, pero no trasciende más allá de la forma estructural. Los aspectos políticos, económicos y culturales terminan relegados a un segundo plano. Por el contrario, para entender por qué una relación social se presenta conformando grupos, comunidades y naciones, es necesario comprender las condiciones culturales que las definen, de forma que se integren discursos que accionen para dicho objetivo.

Segundo, la sociabilidad debe pensarse más allá de la intencionalidad de los sujetos y las asociaciones. Como menciona Bourdieu ${ }^{6}$ (1997, p. 13-21), es en la posición social en un campo, la disposicionalidad o disposición de los individuos en un espacio-tiempo determinado y la toma de posición o elección de los agentes, como puede reflexionarse a unos individuos que viven en un mundo que no controlan totalmente desde sus intenciones, sino que se ubican espacialmente como receptores y productores (simbólicos, económicos, culturales, etc.) con consecuencias no esperadas -o no queridas- $y$ malentendidos que tratan de subsanarse en el grado de comprensión e interrelación, es decir en el habitus o interiorización de lo colectivo en lo individual, o viceversa (Bourdieu, 2013). Es así como puede entenderse el problema que Pilar González menciona en la sociabilidad como objeto de estudio, el que "las 'formas asociativas' no resumen el universo relacional de los actores" (González, 2008, p. 27). Aunque Simmel ya había mencionado que toda socialidad crea en sí misma una insociabilidad -esto frente a la personalidad del individuo que escapa a la asociación-, es aplicable en general a las formas de vida, de ser con los otros, sin limitarse a la asociación, y desde allí poder evaluar los grados de pertenencia de los actores o sujetos.

\section{Conclusión}

De esta manera, el uso de esta categoría debe presentarse más como una forma de investigación concreta y limitada, como una manera de obtener información de la forma social, pero que debe complementarse con el análisis cultural, económico y político que no es un anexo o variable dependiente, sino que compromete de manera clara la organización. Por consiguiente, restarle importancia a las narraciones que constituyen las relaciones entre agentes, sus implicaciones para la continuidad y el cambio, es uno de los problemas que ocurren en estos estudios, ya sea en la reflexión histórica o sociológica, la investigación dependerá de la forma como eje predominante.

$6 \quad$ Pierre Bourdieu, que compartió con nosotros sus observaciones, confirmaría, en lo esencial, los análisis que acabamos de formular, enunciándolos más o menos así: Sí, es necesario estudiar más las asociaciones; sí, la sociabilidad es una noción ambigua, o demasiado compleja, ya que allí se incluyen, a la vez, el estudio de los modos de interacción en la vida cotidiana (cf. La corriente "interaccionista" de la sociología norteamericana) y de las formas de agrupación permanente o modos de "sociación" (de los que la asociación voluntaria es un aspecto); sí, por último, las asociaciones deben clasificarse según criterios menos superficiales que los de la función declarada (y, a fortiori, de la función declarada actual) (Agulhon, 1988/1994, p. 194). 
La sociabilidad, tanto en su propuesta histórica, sociológica y metodológica, debe constituirse como una manera de responder a la pregunta ¿Cómo es posible la sociedad?, sin embargo, al ser resultado en una abstracción teórica, su consecuencia práctica se limita a la expresión relacional de los individuos. Este reconocimiento de sus límites puede tener como consecuencia tanto el mejor aprovechamiento en la teoría, como la pertinencia concreta y definida para cualquier investigación social, puesto que más allá de imponer una visión universal del concepto, define alcances y aplicaciones concretas.

\section{Referencias}

Agulhon, M. (1977). Le Cercle dans la France bourgeoise, 1810-1848, Paris, Armand Colin. (Trad. Agulhon, M. (2009): El círculo burgués, la sociabilidad en Francia 1810-1848. Buenos Aíres, Siglo XIX. DOI: http://dx.doi.org/10.7440/histcrit41.2010.16.

Agulhon, M. (1988/1994). Historia Vagabunda: Etnología y Política en la Francia Contemporánea. México D.F. Instituto de Investigaciones José María Mora.

Bayón, M., Saraví, G. \& Ortega Breña, M. (2013). The Cultural Dimensions of Urban Fragmentation: Segregation, Sociability, and Inequality in Mexico City, Latin American Perspectives 40, 2: 35-52. DOI: 10.1177/0094582X12468865.

Bidart, C. (1988). Sociabilités: quelques variables, Revue française de sociologie 29(4): 621-648. DOI : $10.2307 / 3321515$.

Bourdieu, P. (2013). L'effet Manet, Le Monde Diplomatique 718, pp. 14-15.

Bourdieu, P. (1997). Razones prácticas: sobre la teoría de la acción. Barcelona: Anagrama.

Boutier, J. (1994). Sociabilités locales et réseaux nationaux aux origines de la culture politique démocratique. Les sociétés politiques en Corrèze, 1790-an III. Bulletin de la Société des Lettres, Sciences et Arts de la Corrèze, XCVII: 112-144.

Boutry, P. (1996). Romme et la sociabilité politique révolutionnaire, Annales historiques de la Révolution française 304: 267-282. DOI: 10.3406/ahrf.1996.1972.

Braudel, F. (1953).Georges Gurvitch ou la discontinuité du social, Annales. Économies, Sociétés, Civilisations. 8e année, 3, 347-361. DOI: 10.3406/ahess.1953.2187.

Chapman, W. (2008). Sociabilidades y prácticas políticas en Popayán, 1832-1853, Historia Caribe, 5 (13): 179-207. 
Forse, M. (1981). La sociabilité, Economie et statistique, 132, 39-48. DOI: 10.3406/estat.1981.4476.

Goicovic, I. (2005). Ámbitos de sociabilidad y conflictividad social en Chile tradicional. Siglos XVIII y XIX", Revista Escuela de Historia 1(4): 1-19.

González, P. (1999). Literatura injuriosa y opinión pública en Santiago de Chile durante la primera mitad del siglo XIX", Estudios públicos, 76, 233-262.

González, P. (2008). La sociabilidad y la historia política. Nuevo Mundo Mundos Nuevos [En ligne], Bibliothèque des Auteurs du Centre, González Bernaldo, Pilar, consulté le 09 mai 2014. URL: http://nuevomundo.revues.org/24082 ; DOI: 0.4000/nuevomundo.24082.

Guarín, O. (2010). La sociabilidad política: un juego de luces y sombras. Memoria y Sociedad 14 (29): 25-36.

Guerra, F. (1992). Modernidad e independencias: ensayos sobre las revoluciones hispánicas, Madrid, MAPFRE.

Gurvitch G. (1938/1941). Las formas de la sociabilidad. Buenos Aíres, Losada.

Lash, S. (2003). Lebenssoziologie: Georg Simmel en la era de la información", Estudios sociológicos 21 (3): 523-540.

Loaiza, G. (2011). Sociabilidad, religión y política en la definición de la nación: Colombia 18201886. Bogotá, Colección Bicentenario Universidad Externado de Colombia.

Marqués, E. (2011). "How Do Social Networks Matter in Reducing the Effects of Poverty?" International Journal of Sociology Vol. 41, No. 2: 10-27. DOI: 10.2753/IJS0020-7659410201.

Ponton, R. (1980). Une histoire des sociabilités politiques. Annales. Économies, Sociétés, Civilisations 6, 1269-1280. DOI : 10.3406/ahess.1980.282701.

Simmel, G. (2002a). Cuestiones fundamentales de sociología. Barcelona, Gedisa.

Simmel, G. (1917). Grundfragen der Soziologie: Individumm und Gesellschaft. Berlin, Samlung Göshen. Disponible en: https://archive.org/details/grundfragenderso00simmuoft.

Simmel, G. (200b). Sobre la individualidad y las formas sociales. Buenos Aires, Universidad Nacional de Quilmes. 
Wasserman, S. \& Faust, K. (1994). Social Network Analysis: Methods and Applications. Cambridge, University Press. DOI:http://dx.doi.org/10.1017/CBO9780511815478.003.

Vovelle, M. (1967). Vue nouvelle sur l'histoire des mentalités: La sociabilité méridionale au XVIIle siècle. Revue d'histoire de l'Église de France 53(150): 48-54. DOI: 10.3406/rhef.1967.1770.

Zambrano, F. (1987). Documentos sobre sociabilidad en la Nueva Granada a mediados del siglo XIX. Anuario Colombiano de Historia Social y de la Cultura 15, 323-342. 